\title{
SARS-CoV-2 can infect the placenta and is not associated with specific placental histopathology: a series of 19 placentas from COVID-19-positive mothers
}

\author{
Jonathon L. Hecht ${ }^{1} \cdot$ Bradley Quade $^{2} \cdot$ Vikram Deshpande $^{3} \cdot$ Mari Mino-Kenudson $\mathbb{1}^{3} \cdot$ David T. Ting $\mathbb{D}^{4,5}$. \\ Niyati Desai ${ }^{4} \cdot$ Beata Dygulska $^{6} \cdot$ Taryn Heyman $^{7} \cdot$ Carolyn Salafia $^{8} \cdot$ Dejun Shen $^{9}{ }^{9} \cdot$ Sara V. Bates ${ }^{10}$. \\ Drucilla J. Roberts $\mathbb{1}^{3}$
}

Received: 19 June 2020 / Revised: 24 July 2020 / Accepted: 24 July 2020 / Published online: 2 August 2020

(c) The Author(s), under exclusive licence to United States \& Canadian Academy of Pathology 2020

\begin{abstract}
Congenital infection of SARS-CoV-2 appears to be exceptionally rare despite many cases of COVID-19 during pregnancy. Robust proof of placental infection requires demonstration of viral localization within placental tissue. Only two of the few cases of possible vertical transmission have demonstrated placental infection. None have shown placental expression of the ACE2 or TMPRSS2 protein, both required for viral infection. We examined 19 COVID-19 exposed placentas for histopathologic findings, and for expression of ACE2, and TMPRSS2 by immunohistochemistry. Direct placental SARSCoV-2 expression was studied by two methods-nucleocapsid protein expression by immunohistochemistry, and RNA expression by in situ hybridization. ACE2 membranous expression in the syncytiotrophoblast (ST) of the chorionic villi is predominantly in a polarized pattern with expression highest on the stromal side of the ST. In addition, cytotrophoblast and extravillous trophoblast express ACE2. No ACE2 expression was detected in villous stroma, Hofbauer cells, or endothelial cells. TMPRSS2 expression was only present weakly in the villous endothelium and rarely in the ST. In 2 of 19 cases, SARS-CoV-2 RNA was present in the placenta focally in the ST and cytotrophoblast. There was no characteristic histopathology present in our cases including the two placental infections. We found that the placenta is capable of being infected but that this event is rare. We propose one explanation could be the polarized expression of ACE2 away from the maternal blood and pronounced paucity of TMPRSS2 expression in trophoblast.
\end{abstract}

These authors contributed equally: Jonathon L. Hecht, Bradley Quade

Supplementary information The online version of this article (https:// doi.org/10.1038/s41379-020-0639-4) contains supplementary material, which is available to authorized users.

Drucilla J. Roberts

djroberts@mgh.harvard.edu

1 Department of Pathology, The Beth Israel Deaconess Medical Center, Boston, MA, USA

2 Department of Pathology, Brigham and Women's Hospital, Boston, MA, USA

3 Department of Pathology, The Massachusetts General Hospital, Boston, MA, USA

4 Massachusetts Hospital Cancer Center, Boston, MA, USA

5 Department of Medicine, Massachusetts General Hospital, Boston, MA, USA

\section{Introduction}

SARS-CoV-2 is an enveloped single positive stranded RNA virus responsible for the current pandemic of severe respiratory infections worldwide termed COVID-19 [1]. SARS$\mathrm{CoV}-2$ infects tissues via its receptor, ACE2, and entry into cells requires spike protein cleavage by the serine protease

6 Department of Pediatrics, New York Presbyterian Brooklyn Methodist Hospital, Brooklyn, NY, USA

7 Department of Obstetrics and Gynecology, New York Presbyterian Brooklyn Methodist Hospital, Brooklyn, NY, USA

8 Institute for Basic Research and Developmental Disabilities, Staten Island, NY, USA

9 Department of Pathology, Downey Medical Center, Southern California Permanente Medical Group, Downey, CA, USA

10 Department of Pediatrics, Massachusetts General Hospital, Boston, MA, USA 
TMPRSS2 [2]. Although many reports of COVID-19 in pregnancy describe complications, such as preterm birth [3], vertical transmission is apparently extremely rare [4-10]. Few reports of pregnancy and COVID-19 address placental infection rigorously and, to date, only two reports have demonstrated direct viral infection of the placenta $[9,10]$.

Although the villous syncytiotrophoblast (ST), which lines the surface of the placenta, provides a good barrier to placental infection, viral infections of the placenta do occur, and vertical transmission to the fetus has been demonstrated by many viruses most common are DNA viruses including CMV and HSV [11]. These placental viral infections typically show a histological footprint of chronic inflammation in/of the villi (chronic villitis) or the intervillous space (intervillositis), or both [11]. Infection occurs via either receptors on the villous ST or breaks in the villous trophoblast covering of the villous stroma allowing for infection of stromal cells particularly resident macrophages called Hofbauer cells [12]. Infection of extravillous trophoblast (EVT) has also been demonstrated [13]. Vertical transmission occurs when the virus gains access to fetal blood by direct placental infection or by fetal swallowing or aspiration of infected amniotic fluid.

RNA viruses can also infect the placenta often without causing characteristic histopathology. Exceptions include Hofbauer cell hyperplasia, chronic histiocytic intervillositis, and massive perivillous fibrin deposition (Supplementary Materials, Table 1). To date, a characteristic placental pathology has not been clearly demonstrated in placentas exposed to SARS-CoV-2. Three series that describe the placental pathology of placentas from COVID-19 patients show disparate results but suggest that coagulopathic findings might be common [14-16]. Another three cases have been described in which chronic histiocytic intervillositis was associated with placental infection and apparent vertical transmission $[9,10]$.

The question of whether SARS-CoV-2 infects the placenta is best answered by identifying cases with cellular evidence of viral infection. PCR detection of virus in placental homogenates or swabs is not definitive demonstration of placental infection as they may be contaminated by maternal cells. In addition, demonstration of tissue expression of ACE2 and TMPRSS2 would suggest the capability of the virus to infect the placenta and then possibly the fetus. Herein, we describe 19 placentas from mothers with COVID-19 studied for gross and histopathologic findings, viral expression by in situ hybridization (ISH) and immunohistochemistry (IHC), and ACE2 and TMPRSS2 expression by IHC. We find no specific gross or histopathologic findings in this cohort of COVID-19 exposed placentas. We found two cases of viral infection in the villous trophoblast. We demonstrate that ACE2 is expressed in a membranous pattern in the ST of the chorionic villi. We found that this expression pattern is biased toward the stromal side of the ST, away from the maternal lakes of the placenta. There is also membranous ACE2 expression in the cytotrophoblast (CT) and the EVT. No villous stromal cells, including Hofbauer cells, express ACE2. We demonstrate TMPRSS2 expression is weak and present in the villous endothelium and only rarely in the ST. We propose that these findings explain the limited placental and congenital infection by SARS-CoV-2.

\section{Methods}

\section{Case acquisition}

With institutional review board approval (IRB2020P001116 and IRB2020P001001) and research support agreement (RSA2020A005296), the pathology department databases at the Massachusetts General Hospital (MGH), Brigham and Women's Hospital (BWH), and The Beth Israel Deaconess Medical Center (BIDMC), in Boston, were searched for placentas with the clinical history of "COVID-19-positive" or "coronavirus-positive" from March 1, 2020 to May 15, 2020. Positive cases were then verified by reviewing the clinical history for laboratory values through healthcare records (EHR) at each institution. In total 19 maternal test positives were identified and were included in the study. One negative test was retained as a negative control (MGH3). RNA testing for SARS-CoV-2 was performed on nasopharyngeal swabs using FDA-cleared commercial assays performed according to the manufacturers' instructions.

We used three sets of controls for this study (Table 1). First, although the specificity of our probe for the ISH has been demonstrated in pulmonary tissues [17], we sought to control for its specificity in the placenta. Ten placentas of COVID-19 negative mothers (delivered either before the pandemic or with negative tests) with clinical histories focusing on infections by an RNA virus (one case each of Zika exposure, HIV infection, and Hepatitis $\mathrm{C}$ infection), two cases of intrauterine fetal demise, and with histopathologies associated with congenital infections and coagulopathies (one case each of high stage and grade acute chorioamnionitis, high grade maternal vascular malperfusion (MVM), high grade fetal vascular malperfusion (FVM), high grade villitis of unknown etiology (VUE), chronic histiocytic intervillositis, and multiple intervillous thrombi) were identified from the pathology database using this diagnostic terminology and used as this set of controls. In addition, we used 122 historic "normal" controls from uncomplicated term deliveries (Table 1) to determine the background prevalence of placental histopathologic findings in Boston. These controls were pulled from 2000 to 2004 
Table 1 Controls.

\begin{tabular}{|c|c|c|c|c|}
\hline Control category & $\begin{array}{l}\text { Number of } \\
\text { placentas } \\
\text { examined }\end{array}$ & $\begin{array}{l}\text { Average maternal } \\
\text { age in years (range) }\end{array}$ & $\begin{array}{l}\text { Average gestational age in } \\
\text { weeks + days (range) }\end{array}$ & $\begin{array}{l}\text { Average placental weight } \\
\text { percentile }\{[32], \# 136\} \text { (range) }\end{array}$ \\
\hline For ISH probe specificity & 10 & $31(24-38)$ & $37+5(31+6-40)$ & $\sim 34$ th $(<10 \rightarrow 90)$ \\
\hline $\begin{array}{l}\text { "Normal" controls }=\text { only GBS }+ \text { as } \\
\text { indication for placental examination }\end{array}$ & 122 & $28(16-40)$ & $39+6(37-41+4)$ & $\sim 34$ th $(<10 \rightarrow 90)$ \\
\hline $\begin{array}{l}\text { "Abnormal" controls = placentas from } \\
\text { neonates with clinical diagnosis of HIE }\end{array}$ & 130 & $31(16-44)$ & $39+4(33+6-43)$ & $\sim 30$ th $(<10 \rightarrow 90)$ \\
\hline
\end{tabular}

GBS group B Streptococcus, HIE hypoxic ischemic encephalopathy, ISH in situ hybridization.

and were examined for the sole indication of maternal group B streptococcal (GBS) positivity by clinical history. Finally, we included "pathologic" controls identified in a database of placentas from neonates with a clinical diagnosis of hypoxic ischemic encephalopathy (HIE). This HIE database includes 130 placentas examined over 2 decades and includes late preterm and well as term neonates (Table 1). All control placentas were obtained from the MGH.

\section{Pathologic evaluation}

All placentas were examined for gross and histologic findings at their respective institutions following the Amsterdam Consensus Statement guidelines [18]. Pathologic diagnoses were rendered by experienced perinatal pathologists (DJR, BQ, JLH, CS) following published criteria [11] categorized as shown in Supplementary Materials, Table 2.

\section{Immunohistochemistry and ISH}

Formalin fixed and paraffin embedded (FFPE) blocks from each case and the control were chosen to include membranes, umbilical cord, and full thickness parenchyma. Five-micron serial sections were taken and divided for immunohistochemical (IHC) or ISH studies.

Immunohistochemistry was performed using an automated stainer (Bond-III; Leica Microsystems Bannockburn, IL) with ACE2 Monoclonal Antibody (clone CL4035 [1:15,000], Thermo Fisher Scientific, Waltham, MA), TMPRSS2 antibody (Clone PA5-83286 [1:1,000] ThermoInvitrogen, Carlsbad, CA), and SARS Nucleocapsid Protein Antibody (clone NB100-56576 [1:300], Novus Biologicals, Littleton, $\mathrm{CO}$ ) in accordance with the manufacturer's recommendations.

SARS-CoV-2 RNA ISH was performed using RNAscope $^{\circledR}$ 2.5 LS Probe-V-nCoV2019-S Cat No. 848568 and, RNAscope $^{\circledR}$ 2.5 LS Reagent Kit-RED Cat No. 322150 Advanced Cell Diagnostic (ACD), on automated BondRx platform (Leica Biosystems). Five-micron thick sections of FFPE placental tissues were used including umbilical cord, membranes, and full thickness parenchyma. All the steps from baking for $1 \mathrm{~h}$ at $60^{\circ} \mathrm{C}$ to counterstain with hematoxylin were done on BondRx machine. RNA unmarking is done using Bond Epitope Retrieval Solution 2 for $15 \mathrm{~min}$ at $95^{\circ} \mathrm{C}$ followed by protease treatment for $15 \mathrm{~min}$ and probe hybridization for $2 \mathrm{~h}$. Signal was amplified by series of signal amplification steps followed by color development in red using (Bond Polymer Refine Red Detection, Leica) in the forms of red dots. Specificity of the probe has been previously described [17] but was reinforced with ten placentas from COVID-19 mothers as described above.

\section{Outcomes}

We describe the histopathology of placentas exposed to maternal COVID-19 infections and compare our findings with published prevalence's and the two sets of selected controls. Viral infection of the placenta is examined by RNA and protein expression. Viral receptor and cofactor expression by IHC are provided. Data presented include limited clinical variables.

\section{Role of the funding sources}

The sponsors of this study played no role in study design, data collection, methods, data analysis, data interpretation, or manuscript preparation. The senior author had full access to all the study data and takes final responsibility for the decision for manuscript submission.

\section{Results}

\section{Clinical findings}

Clinical variables are presented in Table 2. Maternal age at delivery averaged 31 years (range 22-42 years, median 32 years) and most were multigravidas (16/19, 84\%). All births except two (MGH2 and MGH11) were singletons. The maternal COVID-19 test was performed peripartum on all cases within an average of 3 days before delivery (range 9 days ante partum-5 days post partum). The gestational 


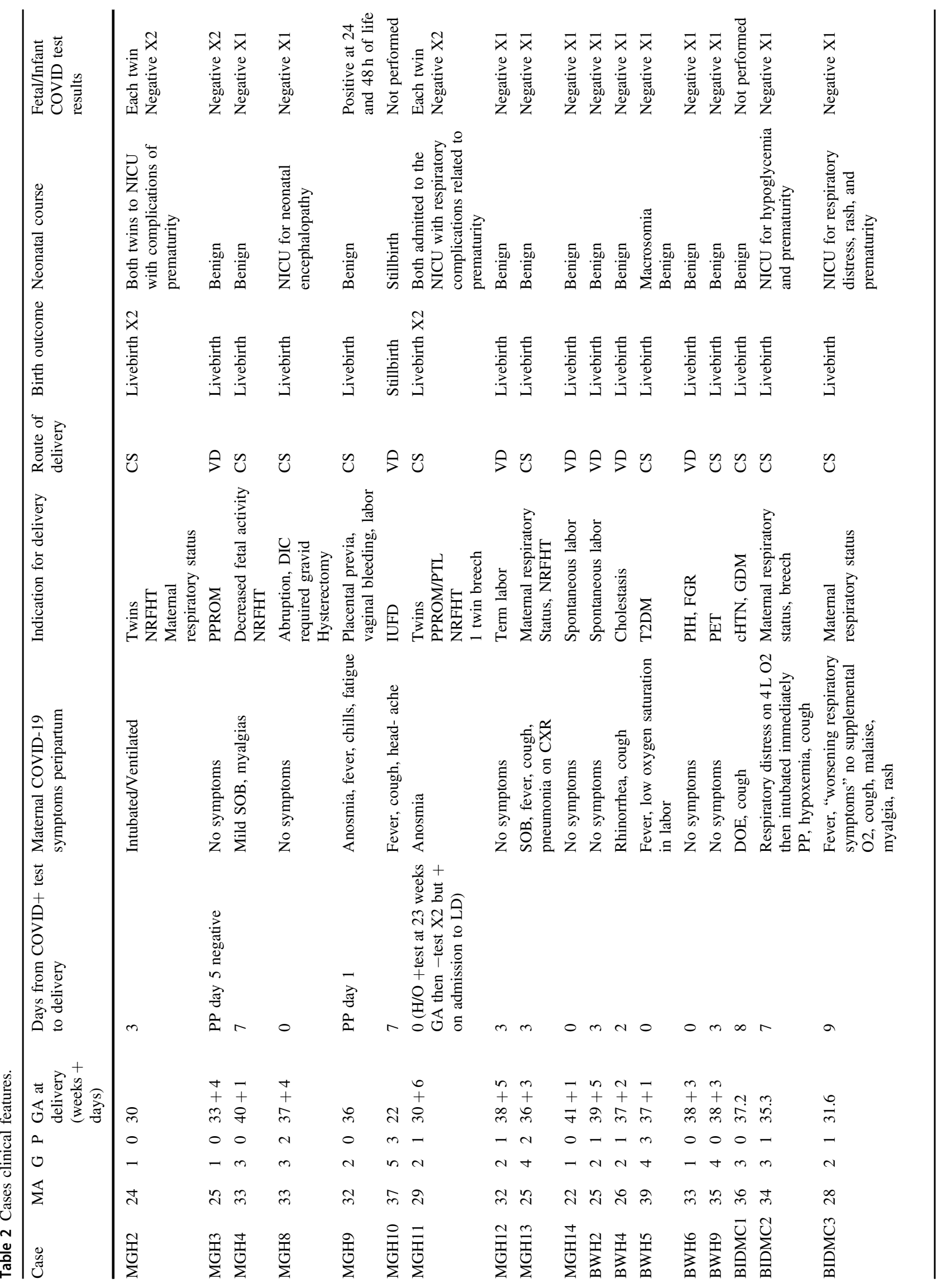




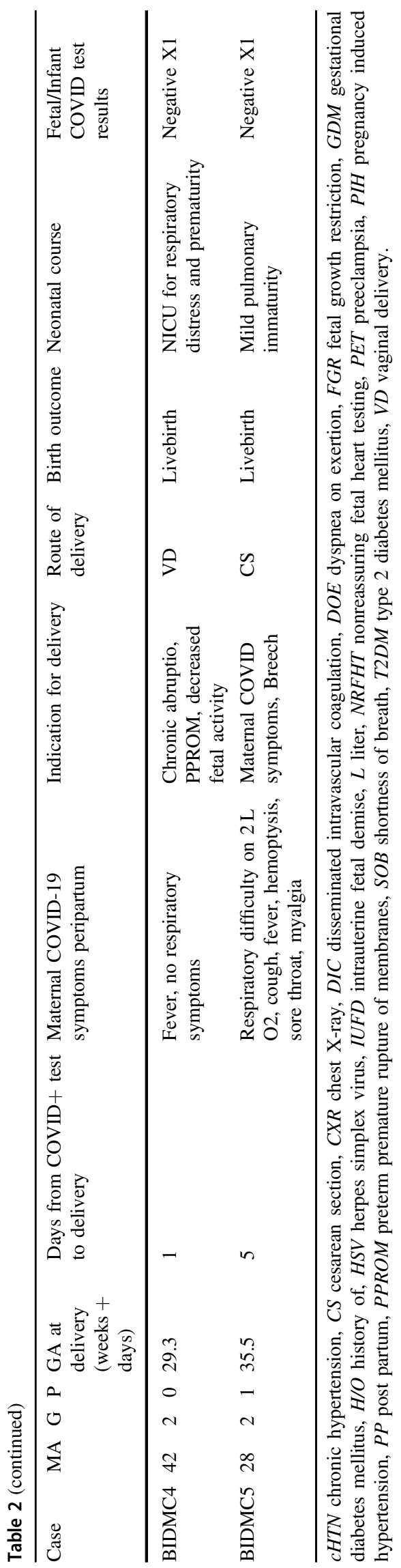

age at delivery averaged 35 3/7 weeks (range 22-41 1 / 7 weeks, median 36 4/7 weeks). Indications for delivery included maternal (spontaneous labor $(n=4)$, respiratory compromise from COVID-19 $(n=6)$, and preeclampsia $(n=3))$, and fetal (abruption $(n=2)$, and nonreassuring fetal testing $(n=4))$. The cesarean delivery rate was $60 \%$ with most performed for fetal indications. Most neonates did well in the early perinatal period except for a few cases with complications of prematurity ( $n=8$ of 21 ). One infant had complications related to intrapartum compromise (MGH8).

The maternal COVID-19 respiratory status was mild except for in five women who had severe symptoms requiring supplemental oxygen, including one who was being mechanically ventilated at the time of delivery (MGH2) and one immediately after delivery (BIDMC2). Only one neonate who was tested, tested positive for SARSCoV-2 24 h (MGH9) [19]. There were no maternal or neonatal deaths in our series, but one (MGH10) was from a 22 weeks gestation intrauterine fetal demise.

\section{Placental gross and histopathology}

Placental pathologic, immunohistochemical, and ISH findings are presented in Table 3. Placental gross and histopathologic features compared with the two control groups are summarized in Supplementary Materials, Table 3. Overall we found no specific gross or histopathology in placentas associated with exposure to SARS-CoV-2 (Table 3). Grossly there were frequent umbilical cord insertional anomalies $(30 \%$, above the background reported prevalence of from 0.1 to $15 \%$ [20]). Looking at the histopathology by category (Supplementary Materials, Table 2), we found any MVM at 25\% (5 of 19 cases), which was similar that present in the "normal" controls $(25 \%$ prevalence) and the "abnormal" controls (15\% prevalence) or a reported background prevalence of 33\% [21]. Three of our MVM cases were low grade and two were high grade (MGH8 and MGH14) neither of which were associated with hypertensive disorders. These two cases had decidual arteriopathy (Fig. 1). Although there were no cases of massive perivillous fibrin deposition, one case had multifocal increased perivillous fibrin but not to the diagnostic criteria of MPFD (MGH14, Fig. 1) [22]. FVM was present in four cases $(20 \%)$ but one was associated with an intrauterine fetal demise (MGH10) and, on review, was considered a postmortem pathology. Excluding this case, the $15 \%$ prevalence of any FVM in our COVID-19 associated placentas is within the published prevalence of FVM at from 7 to $20 \%$ [23]. The case prevalence is higher than the prevalence present in our "normal" controls $(5 \%)$, but similar to that present in our "abnormal" controls (17\%). Thirty percent of cases were classified in the Infe COVID 


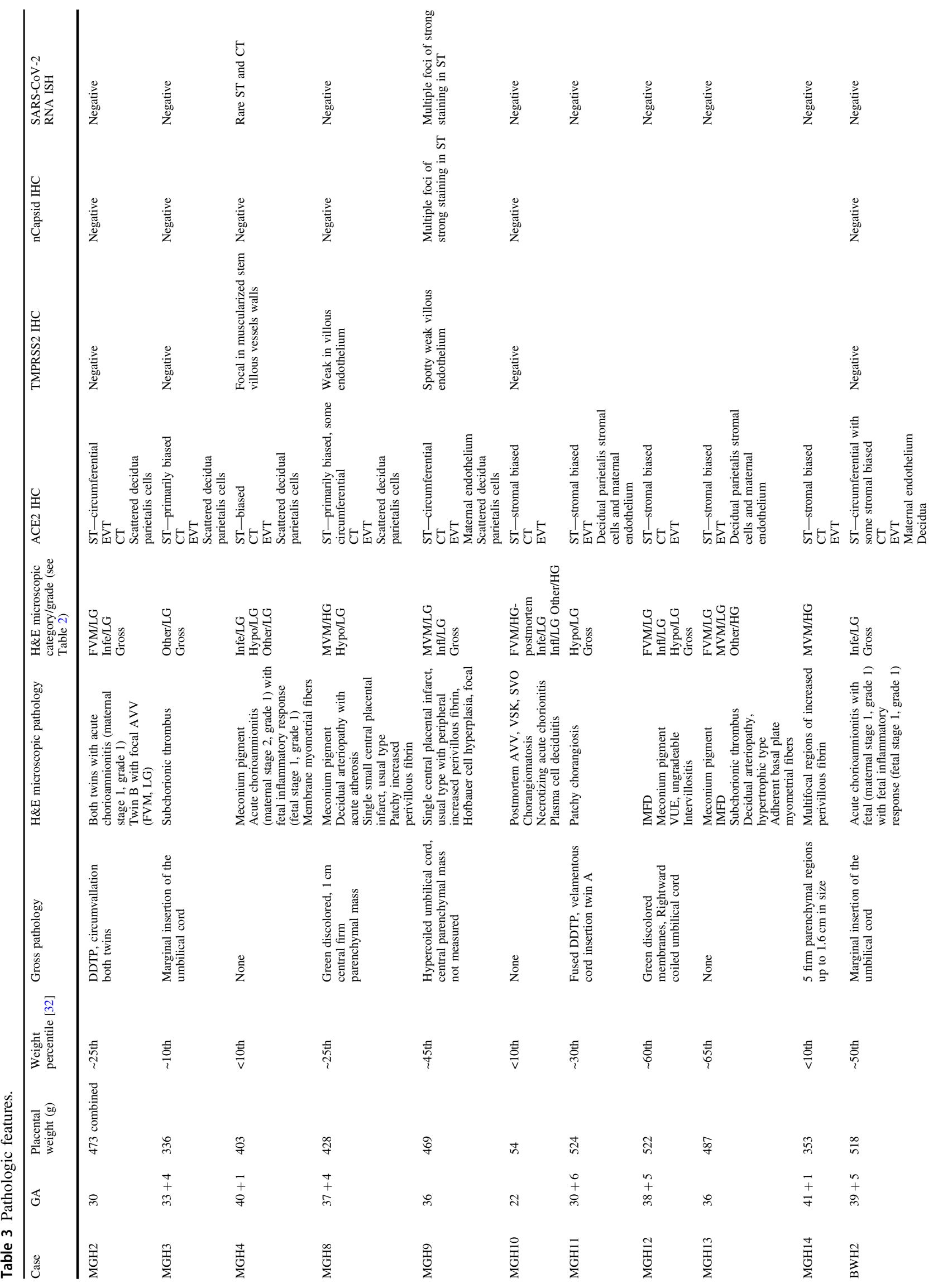




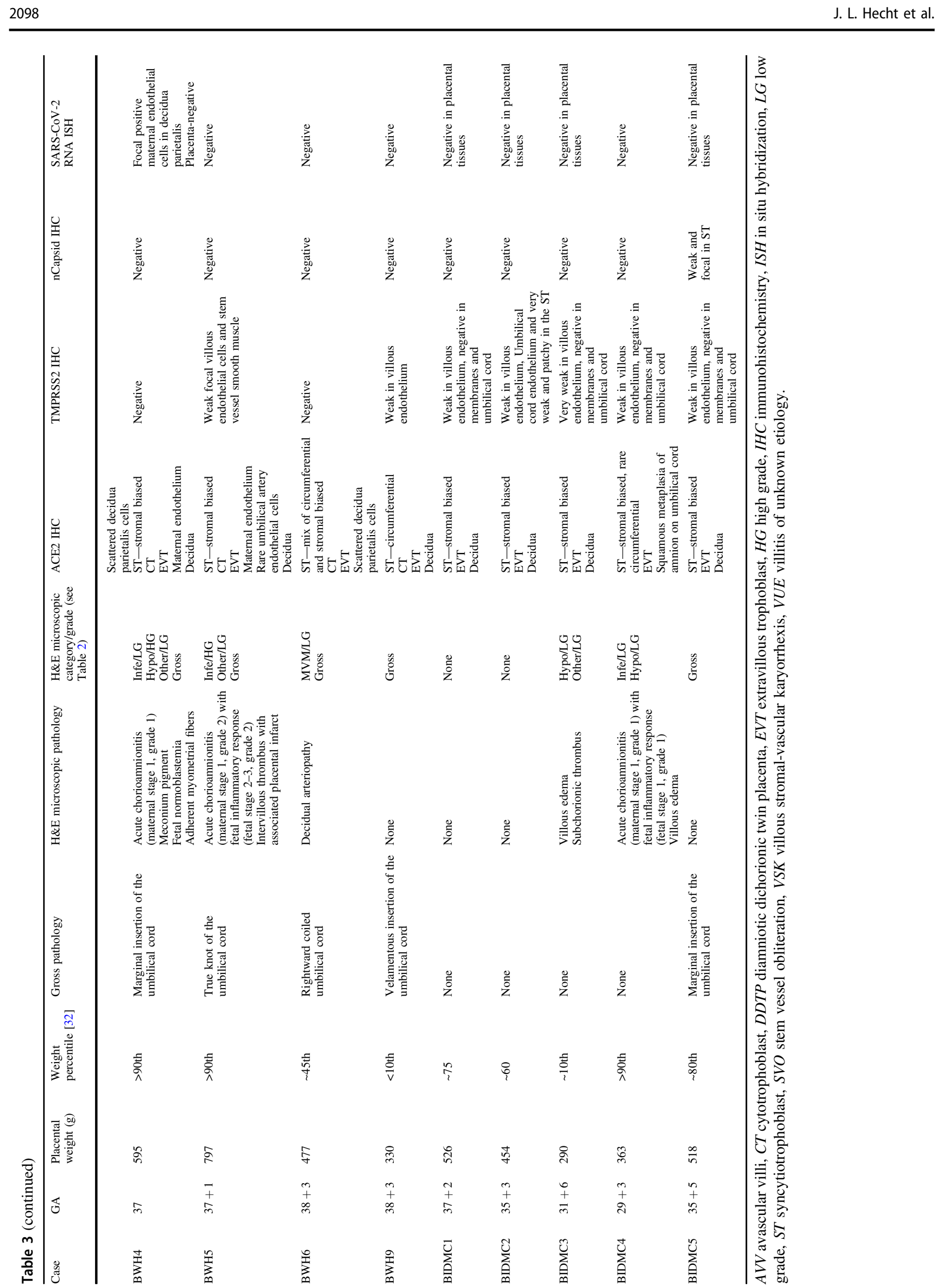

SPRINGER NATURE 
Fig. 1 H\&E Histopathology of COVID-19 exposed placentas. a Decidual arteriopathy with acute atherosis (MGH8), 10X. b Histiocytic intervillositis (MGH12), 40X. c Fetal inflammatory response in the umbilical vein extending into Wharton's jelly (BWH5), 10X. d Increased perivillous fibrin (MGH14), 10X.

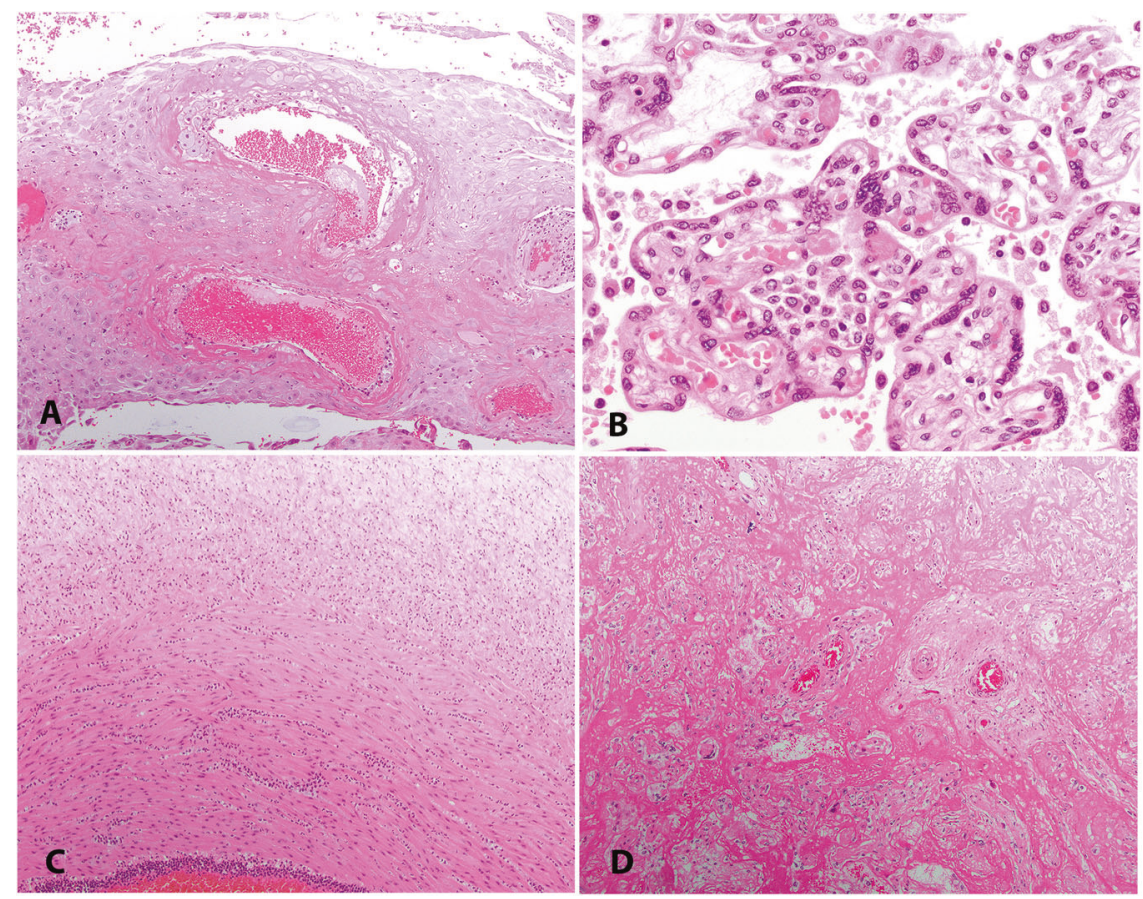

category but only one case was high grade (BWH5, Fig. 1). This prevalence is higher than that seen in our "normal" controls but less than in the "abnormal" controls. All the Infe COVID-19 associated cases were acute chorioamnionitis and four of the sixes cases had a fetal inflammatory response. Inflammatory pathologies were seen in $15 \%$ ( 3 of 19 cases) and all were either low grade or ungradeable. One case had focal Hofbauer cell hyperplasia (MGH9) and one case had histiocytic intervillositis (MGH12, Fig. 1), but not of a degree for a diagnosis of chronic histiocytic intervillositis [24]. There was one case of VUE (5\%), within the published prevalence of 5-15\% [25]. Our $15 \%$ prevalence of any inflammatory pathology compares with any inflammatory pathology in the "normal" controls $(25 / 122,20 \%)$ or the "abnormal" controls $(15 / 130$, $12 \%$ ). If only scoring the Infl-HG placentas, we found 2 in 122 "normal" controls (1.6\%) and 4 in 130 "abnormal" controls (3\%), both above our cases at $0 \%$.

\section{ACE2 expression}

ACE2 was expressed in the ST in a membranous pattern with a polarity such that expression was strongest at the membrane adjacent to the CT and villous stroma (Table 3, Fig. 2). In many cases, the expression was restricted to the stromal side membrane (in 14 of the 20 cases total). ACE2 was also strongly expressed in the villous CT usually in a circumferential membranous pattern and present in a membranous, and occasionally in a cytoplasmic pattern, in scattered EVT in the decidua basalis and chorion laeve (Fig. 2). Maternal vessels showed strong staining present in endothelial cells and pericytes in the decidua parietalis (when present, Fig. 2). No ACE2 staining was present in villous stroma, Hofbauer cells, amniotic epithelium, or fetal endothelium.

\section{TMPRSS2 expression}

In rare cases, TMPRSS2 expression was weakly positive in a cytoplasmic pattern in the villous endothelium (Fig. 2 and Table 3). Only one case had TMPRSS2 staining in a membranous pattern very weakly and in a patchy distribution in the ST (BIDMC2, Fig. 2). Most cases were negative for any detectable expression of TMPRSS2.

\section{Viral expression via IHC or ISH}

Most cases were negative for any viral protein (18 of 19 by IHC) or RNA (17 of 19 by ISH) (Table 3) in the placenta. ISH probe controls were all negative. Rare weak expression was detected by ISH in the maternal endothelium in the decidua parietalis in few cases. One case (MGH9) showed robust expression by both techniques in a patchy distribution in the ST (Fig. 3). One other case (MGH4) had very focal ST and CT expression only by ISH (Fig. 4). Neither showed FVM. MGH9 did show low grade MVM and Infl pathology (Table 3).

\section{Discussion}

Although there have been many reports of infants born to mothers tested to be SARS-CoV-2 positive [3, 7, 26-29], 
Fig. 2 ACE2 and TMPRSS2

expression. Immunohistochemical stains for ACE2 at 60X showing case MGH3 (a, negative control) and case MGH9 (b) show membranous expression of ACE2 in syncytiotrophoblast and

cytotrophoblast. Note the polarity of staining in a such that the strongest staining is on the villous stromal side of the syncytiotrophoblast (arrow) compared to the maternal lake side (arrowhead). This polarity is not present in case MGH9 as stain is present on the maternal lake side (arrowhead) and villous stromal side (arrow) in b. A

syncytiotrophoblastic knot shows cytoplasmic staining in case MGH9 (arrow in b). This finding was focally present in all cases. Immunohistochemical stains for TMPRSS2 at 20X (case MGH3, c) and 40X (case BIDMC2, d). No stain is present in case MGH3 (c, negative control) but weak cytoplasmic staining is preset in the villous endothelium (arrow) and weak membranous staining in the ST (arrowhead) in case BIDMC2 (d). e ACE2 expression in the decidual parietalis maternal endoderm/pericytes (BWH4), 20X. f ACE2 expression in the EVT in the decidua basalis (BIDMC3), 20X.
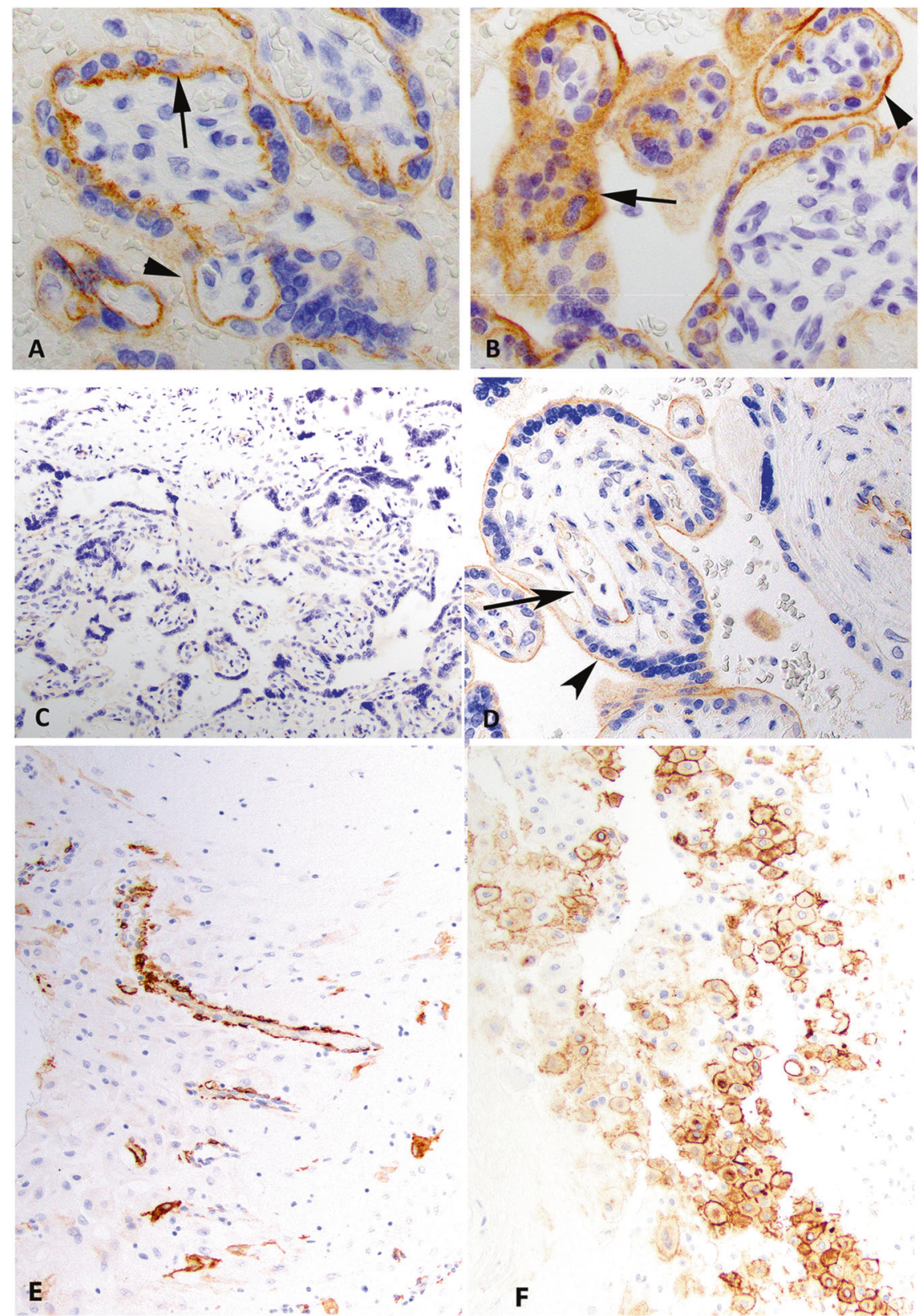

there are only rare case reports of probable vertical transmission $[4,5,9,10,30,31]$. This suggests that SARS-CoV2 either rarely infects the placenta or is prevented from transmission despite placental infection. We show that SARS-CoV-2 can infect the placental ST and CT (Figs. 3 and 4). In addition, we show that ACE2, the receptor for SARS-CoV-2, is expressed in a membranous pattern in the ST and CT of the villi, and in both a membranous and cytoplasmic pattern in the EVT in the chorion laeve and decidua basalis (Fig. 2). We also report a unique polarized expression of ACE2 such that in most cases ST expression is strongest, and sometimes only, present in the cell membrane internal, or stromal, side of the trophoblast (Fig. 2). In addition, we find that TMPRSS2 is rarely expressed in the trophoblast and is expressed only weakly in the villous endothelium (Fig. 2).

Although we had no characteristic placental pathology in our cases from COVID-19 exposed placentas, we did see a spectrum of pathologies (Table 3). Interestingly we saw only one case each of chronic villitis, intervillositis, and increased perivillous fibrin. Except for an increase in gross pathologies, the prevalence of the other categories was within our control groups and published series. The gross pathology findings are difficult to explain but are unlikely to be related to SARS-CoV-2 exposure.

To the authors knowledge, there are seven reports of placental findings related to SARS-CoV-2 exposure (Supplementary Materials, Table 1). In Baergen and Heller's 
Fig. 3 Viral expression via IHC and ISH. Left column of images from $\mathrm{MGH} 3$ (negative control). Right column of images from MGH9. H\&E (a, b), immunohistochemical stains for nCapsid (c, d), and in situ hybridization for viral RNA (e, f), 20X.
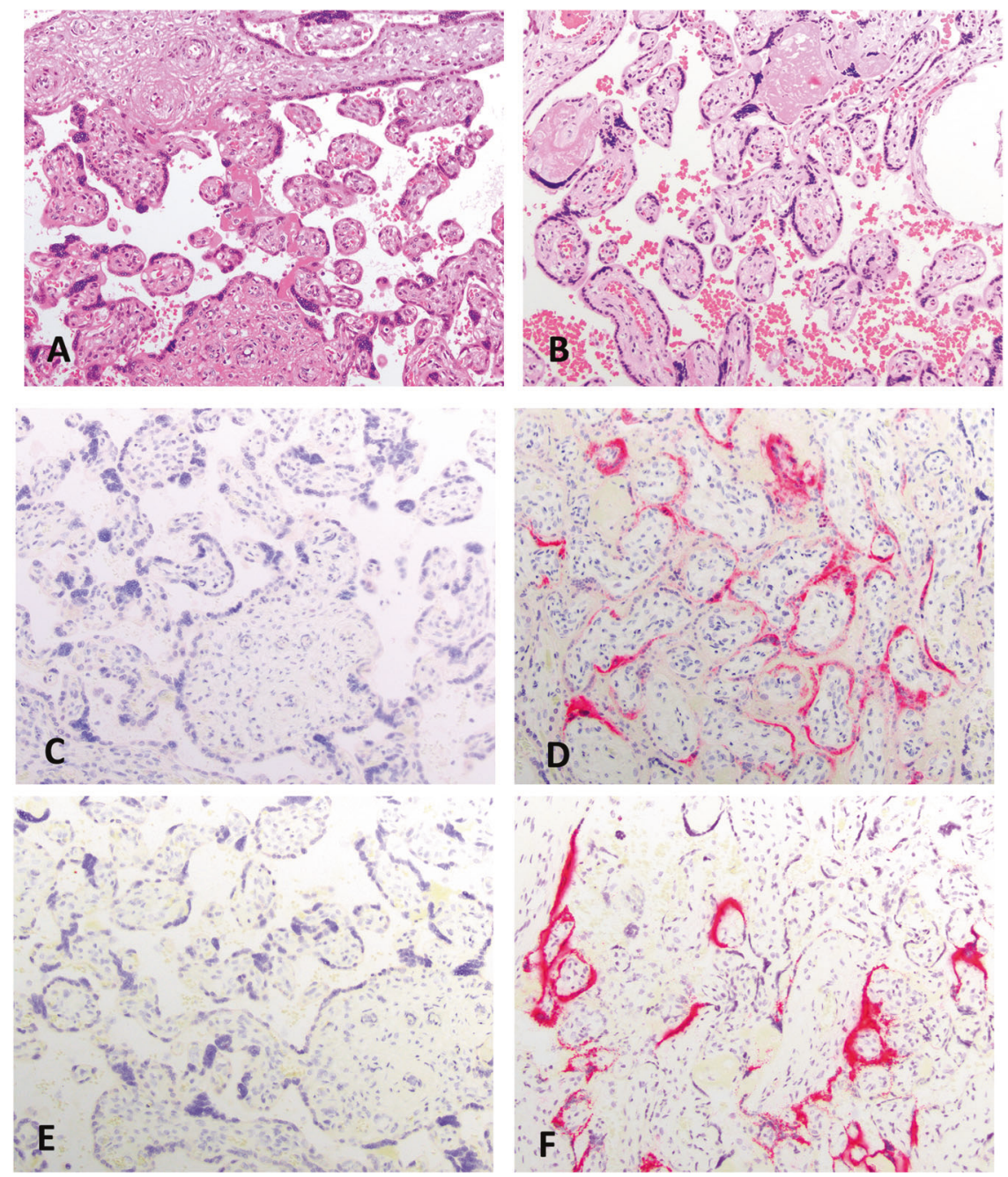

report [14], they that found 10 of 20 exposed placentas had FVM. Although not graded in their report, by description it is likely that four were high grade. Our prevalence of $15 \%$ of FVM (all low grade) is higher than our "normal" controls and this may be a real increase, similar to prevalence in our "abnormal" controls. The manuscript by Shanes et al. [16] describes an increased prevalence of MVM in their series of 16 placentas delivered to women with COVID-19. Although there were only two cases with MVM in their series of 16, which was significantly above their robust controls. There is one manuscript [15] that describes the placentas from three COVID-19 infected mothers in China. All three placentas were described as having increased perivillous fibrin. Baud et al. [4] published a case of apparent placenta infection in a 19 weeks intrauterine fetal demise. The infection was detected by placental homogenate and swab PCR (which we believe may be contaminated by maternal tissue). Fetal tissues were negative for SARS-CoV-2. The figures demonstrate mild acute chorioamnionitis with a fetal inflammatory response. Although the authors conclude that there was increased perivillous fibrin and syncytial knots (both features of MVM), the image is not convincing. In our opinion, the findings are more consistent with the effects of retention of an intrauterine fetal demise. Our series found MVM in 25\% of cases but that was within our control prevalence. Kirtsman et al. [5] described CHI in a placenta from a case of potential vertical transmission. Three of these seven reports found features that suggest a coagulopathic process. Although we did see MVM and FVM in our series, we did not see them increased over published prevalence's or our two sets of controls. Two recent case reports describe placental infection by ISH in three cases [10] and in one case [9]. Both of these reports found infection in the ST as did we. Hosier et al. [9] also demonstrated virus in the ST by electron microscopy. Three of these four cases demonstrated histiocytic intervillositis, as we saw in one of our cases, but not in either of our two cases with infection. We believe that our data and the published literature has not definitively identified a histopathologic footprint of SARS$\mathrm{CoV}-2$ infection of the placenta or features characteristic of exposure without infection. 
Fig. 4 Viral expression via ISH in MGH4. Focal expression in the syncytiotrophoblast and cytotrophoblast at 40X (left panel) and 60X (right panel).

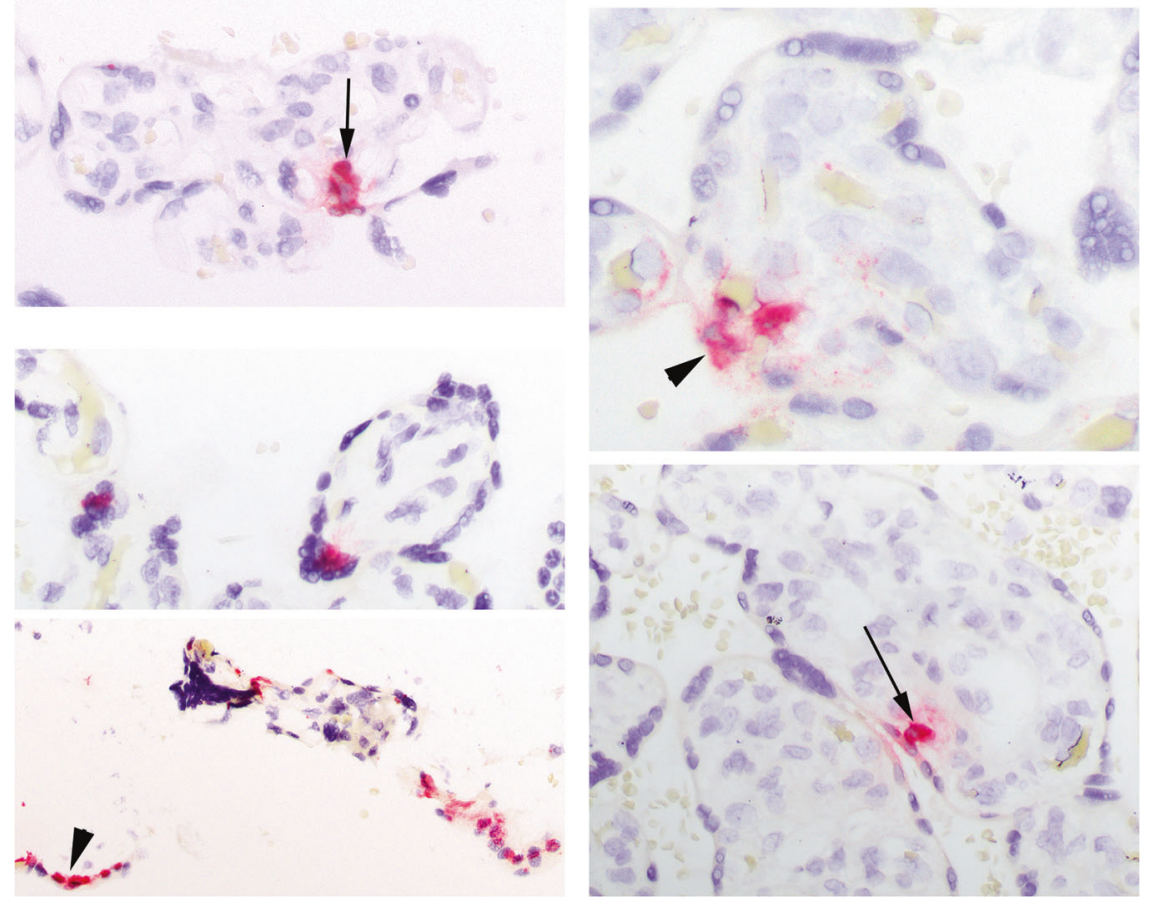

We expected an increase in chronic inflammatory pathologies, particularly chronic villitis or chronic intervillositis, in these exposed placentas given what has been reported in other RNA viral infections (Supplementary Materials, Table 1). We did not see this in our cases. In our series, there are three cases with a chronic placental inflammatory process, but none are high grade. Although this represents a prevalence of $15 \%$ overall, there was only one case of chronic villitis (VUE, ungradeable) at 5\%, within the accepted background prevalence published at from 5 to $15 \%$ [25]. In the published literature, there are only case reports identifying a chronic inflammatory process, three identifying intervillositis $[5,9,10]$. Intervillositis was seen in one of our cases (and was not one of the two cases with demonstrable placental infection) but has not been described in other series $[14,16]$. Whether it is a true marker for SAR-CoV-2 placental infection needs more study.

Our morphologic study is limited in that we did not use optimal controls. We believe that the best controls would have been placentas delivered to women with COVID-19 symptoms (e.g., hypoxia) but SARS-Cov-2 test negative. This should be done to definitively identify if coagulopathic or inflammatory pathologies are increased in this population. In our one placenta from a symptomatic but COVID19 test negative woman, the placenta showed a subchorionic thrombus (MGH3).

In summary, we show that ACE2 is expressed in the trophoblast of the placenta with a polarized membranous pattern in the ST. We also show that TMPRSS2 is expressed weakly mainly in the chorionic villous endothelium. We demonstrate SARS-CoV-2 virus infection of the ST and CT. This is conclusive evidence of placental infection and therefore confirms the possibility of vertical transmission. As we have no definitive cases of congenital infection, these data suggest that placental infection may not always equate with vertical transmission.

Acknowledgements We would like to acknowledge the incredible assistance of the histology and immunohistochemistry laboratories at the MGH and Linda Arini for administrative assistance. We would like to dedicate this manuscript to those mothers and babies affected by the COVID-19 pandemic.

Author contributions All authors had full access to the data in this report and take responsibility for its integrity and accuracy. JLH, BQ, and DJR were responsible for the concept and analysis of this study. $\mathrm{BD}, \mathrm{TH}, \mathrm{CS}$, and DS contributed cases and reports. The HIE database is maintained by SVB. Authors JLH, BQ, and DJR were responsible for drafting this manuscript but all authors had input into the final version. DTT, VD, and ND were responsible for the ISH studies. MM-K was responsible for optimizing the IHC studies.

\section{Compliance with ethical standards}

Conflict of interest DTT has received consulting fees from Merrimack Pharmaceuticals, Ventana Roche, Foundation Medicine, Inc., and EMD Millipore Sigma, which are not related to this work. DTT is a founder and has equity in PanTher Therapeutics and TellBio, Inc., which is not related to this work. DTT is cofounder and own equity in ROME Therapeutics, which is not related to this work. Parts of this work were supported by ACD-Biotechne (ND, VD, DTT). DTT's 
interests were reviewed and are managed by Mass General Brigham in accordance with their conflict of interest policies. MM-K has received consulting fees from $\mathrm{H} 3$ Biomedicine and AstraZeneca and institutional grant support from Novartis, all of which are not related to this work. DJR received royalties from UpToDate and Cambridge University Press as an author, not related to this work. All other authors declare no competing interests.

Publisher's note Springer Nature remains neutral with regard to jurisdictional claims in published maps and institutional affiliations.

\section{References}

1. Zhu N, Zhang D, Wang W, Li X, Yang B, Song J, et al. A novel coronavirus from patients with pneumonia in China, 2019. N Engl J Med. 2020;382:727-33.

2. Hoffmann M, Kleine-Weber H, Schroeder S, Kruger N, Herrler T, Erichsen S, et al. SARS-CoV-2 cell entry depends on ACE2 and TMPRSS2 and is blocked by a clinically proven protease inhibitor. Cell. 2020;181:e8.

3. Mullins E, Evans D, Viner RM, O'Brien P, Morris E. Coronavirus in pregnancy and delivery: rapid review. Ultrasound Obstet Gynecol. 2020;55:586-92.

4. Baud D, Greub G, Favre G, Gengler C, Jaton K, Dubruc E, et al. Second-trimester miscarriage in a pregnant woman with SARSCoV-2 infection. JAMA. 2020. https://doi.org/10.1001/jama. 2020.7233.

5. Kirtsman M, Diambomba Y, Poutanen SM, Malinowski AK, Vlachodimitropoulou E, Parks WT, et al. Probable congenital SARS-CoV-2 infection in a neonate born to a woman with active SARS-CoV-2 infection. CMAJ. 2020;192:E647-50.

6. Chen S, Huang B, Luo DJ, Li X, Yang F, Zhao Y, et al. [Pregnant women with new coronavirus infection: a clinical characteristics and placental pathological analysis of three cases]. Zhonghua Bing Li Xue Za Zhi. 2020;49:E005.

7. Liu Y, Chen H, Tang K, Guo Y. Clinical manifestations and outcome of SARS-CoV-2 infection during pregnancy. J Infect. 2020. https://doi.org/10.1016/j.inf.2020.02.028.

8. Zhu H, Wang L, Fang C, Peng S, Zhang L, Chang G, et al. Clinical analysis of 10 neonates born to mothers with 2019-nCoV pneumonia. Transl Pediatr. 2020;9:51-60.

9. Hosier H, Farhadian R, Morotti R, Deshmukh U, Lu-Culligan A, Campbell K, et al. SARS-CoV-2 infection of the placenta. 2020. https://doi.org/10.1172/JCI139569.

10. Patane L, Morotti D, Giunta MR, Sigismondi C, Piccoli MG, Frigerio L, et al. Vertical transmissioin of coronavirus disease 2019: severe acute respiratory syndrome coronavirus 2 RNA on the fetal side of the placenta in pregnancies with COVID-19positive mothers and neonates at birth. AJOG MFM. 2020. https:// doi.org/10.1016/j.ajogmf.2020.100145.

11. Redline RW, Boyd TK, Roberts DJ, editors. Placental and gestational pathology. Vol 1. Cambridge, UK: Cambridge University Place; 2018. p. 344.

12. Parry S, Holder J, Strauss JF 3rd. Mechanisms of trophoblastvirus interaction. J Reprod Immunol. 1997;37:25-34.

13. Koi H, Zhang J, Parry S. The mechanisms of placental viral infection. Ann N Y Acad Sci. 2001;943:148-56.

14. Baergen RN, Heller DS. Placental pathology in Covid-19 positive mothers: preliminary findings. Pediatr Dev Pathol. 2020;23:177-80.

15. Shuo C, Bo H, Danju L, Xiang L, Fan Y, Yin Z, et al. Clinical characteristics and pathological analysis of placenta in three cases of pregnant women with novel coronavirus infection. Chin J Pathol. 2020;49:418-23.

16. Shanes ED, Mithal LB, Otero S, Azad HA, Miller ES, Goldstein JA. Placental pathology in COVID-19. Am J Clin Pathol. 2020;154:23-32.

17. Massoth LR, Desai N, Szabolcs A, Harris CK, Neyaz A, Crotty $\mathrm{R}$, et al. Comparison of RNA in-situ hybridization and immunohistochmistry techniques for the detection and localization of SARS-CoV-2 in human tissues. Am J Surg Pathol. 2020. In press.

18. Khong TY, Mooney EE, Ariel I, Balmus NC, Boyd TK, Brundler MA, et al. Sampling and definitions of placental lesions: Amsterdam Placental Workshop Group Consensus Statement. Arch Pathol Lab Med. 2016;140:698-713.

19. Alamar I, Heyman T, Abu-Arja M, Zhang P, Salafia CM, Roberts DJ, et al. A SARS-COV-2 positive triad: mother, placenta and newborn. J Ped Infect Dis Soc. Under review.

20. Ebbing C, Kiserud T, Johnsen SL, Albrechtsen S, Rasmussen S. Prevalence, risk factors and outcomes of velamentous and marginal cord insertions: a population-based study of 634,741 pregnancies. PLoS ONE. 2013;8:e70380.

21. Catov JM, Muldoon MF, Reis SE, Ness RB, Nguyen LN, Yamal JM, et al. Preterm birth with placental evidence of malperfusion is associated with cardiovascular risk factors after pregnancy: a prospective cohort study. BJOG. 2017;125:1009-17.

22. Faye-Petersen OM, Ernst LM. Maternal floor infarction and massive perivillous fibrin deposition. Surg Pathol Clin. 2013;6:101-14.

23. Vik T, Redline R, Nelson KB, Bjellmo S, Vogt C, Ng P, et al. The placenta in neonatal encephalopathy: a case-control study. J Pediatr. 2018;202:e3.

24. Bos M, Nikkels PGJ, Cohen D, Schoones JW, Bloemenkamp KWM, Bruijn JA, et al. Towards standardized criteria for diagnosing chronic intervillositis of unknown etiology: a systematic review. Placenta. 2018;61:80-8.

25. Redline RW. Villitis of unknown etiology: noninfectious chronic villitis in the placenta. Hum Pathol. 2007;38:1439-46.

26. Yu N, Li W, Kang Q, Xiong Z, Wang S, Lin X, et al. Clinical features and obstetric and neonatal outcomes of pregnant patients with COVID-19 in Wuhan, China: a retrospective, single-centre, descriptive study. Lancet Infect Dis. 2020;20:559-64.

27. Yan J, Guo J, Fan C, Juan J, Yu X, Li J, et al. Coronavirus disease 2019 (COVID-19) in pregnant women: a report based on 116 cases. Am J Obstet Gynecol. 2020;223:111e1-14.

28. Schwartz DA, Dhaliwal A. Infections in pregnancy with covid-19 and other respiratory rna virus diseases are rarely, if ever, transmitted to the fetus: experiences with Coronaviruses, HPIV, hMPV, RSV, and Influenza A. Arch Pathol Lab Med. 2020. https://doi.org/10.5858/arpa.2020-0211-SA.

29. Liu D, Li L, Wu X, Zheng D, Wang J, Yang L, et al. Pregnancy and perinatal outcomes of women with coronavirus disease (COVID-19) pneumonia: a preliminary analysis. Am J Roentgenol. 2020;215:1-6.

30. Dong L, Tian J, He S, Zhu C, Wang J, Liu C, et al. Possible vertical transmission of SARS-CoV-2 from an infected mother to her newborn. JAMA. 2020. https://doi.org/10.1001/jama.2020. 4621.

31. Zeng H, Xu C, Fan J, Tang Y, Deng Q, Zhang W, et al. Antibodies in infants born to mothers with COVID-19 pneumonia. JAMA. 2020. https://doi.org/10.1001/jama.2020.4861.

32. Pinar H, Sung CJ, Oyer CE, Singer DB. Reference values for singleton and twin placental weights. Pediatr Pathol Lab Med. 1996;16:901-7. 